\title{
Statistical Characterisation of the EM Interferences Acting on GSM-R Antennas Fixed Above Moving Trains
}

\author{
N. Ben Slimen*, V. Deniau*, J. Rioult ${ }^{*}$, S. Dudoyer* and S. Baranowski** \\ *INRETS-LEOST, 20 rue Elisée reclus 59650 Villeneuve d'Ascq, France, last-name@ inrets.fr \\ **IEMN-TELICE, USTL Bat P3 Villeneuve d'Ascq, France, sylvie.baranowski@univ-lille1.fr
}

\begin{abstract}
Shortened version of the title: Statistical characterisation of the transient EM noise in railway context
\end{abstract}

\begin{abstract}
In order to harmonise the communication systems between the train and the railway control centres over the European territory, a GSM-R (Global System for Mobile communications - Railways) communication network is progressively deployed along the European railway network. However, the GSM-R communications on board high speed trains can be disturbed by the transient electromagnetic (EM) disturbances induced by the sliding contact between the catenary and the pantograph. In order to study the immunity of the embedded GSM-R communication system against these transient electromagnetic disturbances, the transient interferences induced on the GSM-R antennas on board trains, were characterised in terms of time and amplitude parameters. Measurement campaigns were carried out in France to collect a large number of induced EM interferences on GSM-R antennas fixed on the train roof. With the ultimate goal of generating transient noise scenarios representative of those detected by the antennas, and performing immunity tests in laboratory, statistical distributions of the characteristics (rise time, time duration, repetition rate, amplitude) of the transients are presented. This paper presents the different steps of the analysis of the transient disturbances and the generation of the transient EM scenarios.
\end{abstract}

\section{Introduction}

To ensure the interoperability of trains in Europe, the GSM-R communication system will be progressively deployed over all the railway network of the European Union. It is important to notice that the GSM-R is not similar to the GSM system but the use of the same communication protocol.

The GSM-R is employed to guarantee the transmission of voice and signalling data between the train and the control centres by means of a mobile transmitter-receiver (mobile with respect to base stations but fixed inside the locomotive) connected to a GSM-R antenna fixed on the roof of the locomotive and base stations installed in the neighbourhood of the rail track. A shielded cable ensures the connexion between the GSM-R antenna and the mobile transmitter- receiver. Traffic data and voice collected throughout the GSM-R system are sent to the train driver.

The reliability of the GSM-R system became an important parameter for the safety and the good operation of railway traffic. The signals received by the GSM-R antennas fixed on board the trains can be affected by transient electromagnetic emissions finding their source in an imperfect sliding contact between the catenary and the pantograph. Under some particular conditions, transient events can be observable with the naked eye as sparks. These EM transient events are penalizing for the embedded telecommunication systems, since they are broadband signals and can generate high levels of noise in the GSM-R frequency bands. In addition, the GSM-R is a digital communication system and there is no EMC standard well suited to study the EM immunity of this system against transient EM events.

In this context, the immunity of the GSM-R system against the transient electromagnetic noises observed on board moving trains has to be studied carefully.

Generally, the immunity of electric or electronic systems is defined as the ability of a device to perform in nominal operating conditions without degradation in the presence of standardized EM disturbances. The EM immunity standards describe the test configuration and define the time, frequency and amplitude characteristics of permanent and transient EM noises applied on the equipment under test (EUT). The tests are performed in EMC test facilities. The immunity of the EUT is verified if no failure is observed during and after the test. The EMC standards dealing with the immunity tests are adapted to electronic systems but are applicable with difficulty for digital communication systems. Our main objectives in this paper are to present and test the feasibility of a new method to characterize the EM immunity of the GSM-R system against the transient EM noise observed on board moving trains. This method is based on the statistical characterisation of some parameters of the transient EM noise observed through a GSM-R antenna fixed above a moving train.

The digital communication systems use various communication protocols which combines different corrector codes. The corrector codes are used to correct some disturbed bits over the transmitted bursts. The immunity test methods have to be adapted to permit testing of the robustness of such complex systems against EM disturbances and to analyze relevant immunity criteria.

The GSM-R uses different frequency channels over a given time. Thus, we will present in detail the temporal values that 
characterise the GSM-R protocol and which can be confronted with the temporal characteristics of transient noises. Then, a precise analysis of the time characteristics of the transient disturbances collected during measurement campaigns will be carried out. Statistical distributions of the different parameters which define the transient EM disturbances will be extracted. We will then propose a method to generate transient noise scenarios in laboratory, using the statistical distributions. Finally the immunity of the GSM-R system to EM noise conditions identical to those being on the train roof could be evaluated in laboratory.

\section{Time characteristics of the GSM-R}

The GSM-R system [1] is based on the standard GSM Phase $2+$ in addition to some advanced functions specifically developed for railway applications. Moreover, the GSM-R system employs two specific frequency bands [921-925] MHz for the downlink (from the control centres to the train) and [876-880] $\mathrm{MHz}$ for the uplink (from the train to the control centres). Each frequency band is divided into 20 frequency channels of $200 \mathrm{kHz}$. For security purposes, the two bound channels of each frequency band are not used.

The GSM-R is a TDMA (Time Division Multiple Access) system. Thus, for each frequency channel, data are organized per periodic TDMA frame, with a period of $4.615 \mathrm{~ms}$. Each TDMA frame is divided into 8 time intervals of $577 \mu$ s called "Time Slots". Therefore, each user occupies a frequency channel only one eighth of the time and by period of $577 \mu \mathrm{s}$. Each "Time Slot" includes 156 bits. The transmission time of one bit is $3.7 \mu \mathrm{s}$. Figure 1 provides a schematic representation of the GSM-R signal which is emitted during a "time slot" [2].

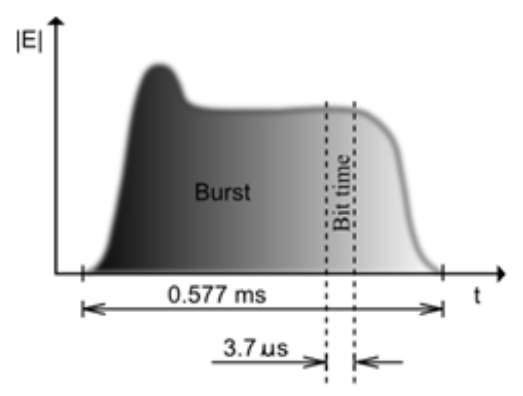

Figure 1: Time characteristics of one GSM-R Burst

\section{Definition of the time characteristics of the transient EM interferences}

Generally, the immunity of electronic and electrical systems which the access ports (power, signals or control) are confronted to fast transient burst coming mainly from the inductive load disconnection, bouncing power relays...etc. is studied according to the recommendations described in the EN 61000-4-4 standard [3].

The standard concerns the application of transient test disturbances at each different ports of Equipment Under Test
(EUT), using a capacitive coupling device over the different lines connected to the EUT. Dealing with telecommunication equipments, a part of the test described in this standard focus on the effect of the disturbances affecting the input and output telecommunication signal ports.

This standard is also the reference in the ETSI standards [4] for testing electromagnetic immunity of telecommunication network equipment.

In the EN 61000-4-4 standard, the transient EM test signals which are intentionally generated to control the immunity of a device are notably defined in terms of maximal amplitude $(\boldsymbol{A})$ and corresponding time interval $(\boldsymbol{T I})$, rise time $(\boldsymbol{R} \boldsymbol{T})$ and time duration $(\boldsymbol{T D})$. The maximal amplitude $\boldsymbol{A}$ is the peak amplitude of the transient.

The $\boldsymbol{T D}$ presented Figure 2a, corresponds to the time duration when the EM noise level of the transient event is higher than $50 \%$ of its peak amplitude A. The $\boldsymbol{R} \boldsymbol{T}$ is defined as the time between $10 \%$ and $90 \%$ of $\boldsymbol{A}$ Figure 2b. The time interval $\boldsymbol{T I}$ is the time between two successive transients Figure 2c.

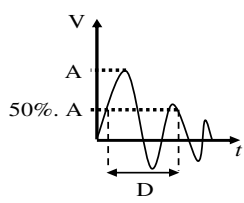

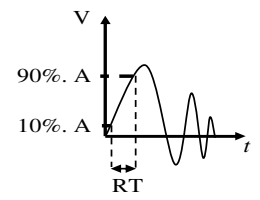

(c)

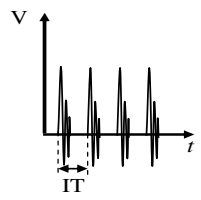

Figure 2: Definitions of the time characteristics of transient signals in EN 61000-4-4

The standardized values for each transient parameter described above given in the 61000-4-4 standard are as follows:

$\boldsymbol{R T}: 5 \mathrm{~ns} \pm 30 \%, \boldsymbol{T D}: 50 \mathrm{~ns} \pm 30 \%, \boldsymbol{T I}:(0.2 \mathrm{~ms}$ or $0.4 \mathrm{~ms})$ according to the peak Amplitude A of the transients, where $\boldsymbol{A}$ remains constant for each test signal.

These different characteristics have an important influence on the frequency bandwidth covered by the transient. However, the values of the time parameters indicated in the 61000-4-4 standard are not necessarily representative of the transient disturbances received by the GSM-R antenna on board the train. Moreover, a transient with a $5 \mathrm{~ns}$ rise time will not necessarily cover the GSM-R frequency bands. We have then decided to focus on the characterisation of the real time parameters of the on-board EM transients in order to compare them with the time characteristics of the GSM-R protocol [5]. The characterisation of the transient events observed on board moving trains was performed according to the definitions of the rise time, time duration, amplitude, and time interval of the transient events given in the 61000-4-4 standard.

\section{Measurement campaign configuration}

The results presented in this paper are extracted from the transient events measured on a train including one locomotive and eight cars, as presented in Figure 3.

Measurements were carried out in France between Saint Pierre des Corps (SPC) near the city of Tours and Nantes. The 
train path distance was about $200 \mathrm{~km}$ with a $25,000 \mathrm{~V}$ AC (50 $\mathrm{Hz}$ ) supply voltage. The cruising speed of the train was about $160 \mathrm{~km} / \mathrm{h}$ and the maximal speed was $200 \mathrm{~km} / \mathrm{h}$. In order to record the EM noise observed on the roof of the train, the measurements were performed with a digital oscilloscope connected to a GSM-R antenna fixed on the top of the fourth car, at approximately $80 \mathrm{~m}$ from the pantograph. The statistical results presented in this paper are obtained from the measurements performed during a round trip between Nantes and SPC.

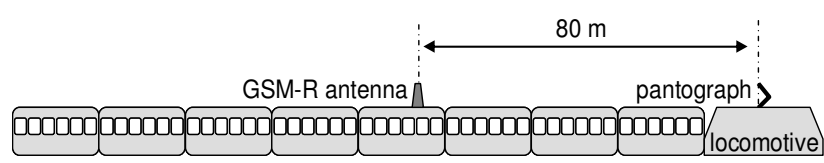

Figure 3: Measurement configuration on board the train

The GSM-R antenna is a bi-band antenna working in the frequency band of the French railway radio system [420-520] $\mathrm{MHz}$ and of the GSM-R system [820-1000] MHz.

Our objective being to extract the time characteristics of the EM transient events, experiments were carried out using a digital oscilloscope with a $20 \mathrm{GHz}$ sampling frequency. The detection of transient signals was dependent on the triggering level of the oscilloscope. Generally, the trigger level is fixed arbitrarily by the operator at a value barely higher than the permanent noise level observed at low speed (less than $60 \mathrm{~km} / \mathrm{h}$ ).

Each transient event detected during the train path was recorded in a $200 \mathrm{~ns}$ time window. During about 100 minutes of measurement, the oscilloscope collected about 25,700 transient signals. The randomness of the characteristics of the transient signals led us to perform a statistical analysis. Statistical studies on the different time characteristics and amplitude of the collected transients are presented in the next part.

\section{Statistical study on the time characteristics and amplitude of the transient events}

Statistical studies presented in this paper were performed with the distribution fitting tool of MATLAB and the analytical probability density functions used were extracted from [6].

The study presented below does not aim to establish a link between the different characteristics of the transient events and the physical mechanisms that could control the generation of the transients.

In fact, it is obviously difficult to perform measurement campaigns where we can control all the operating conditions, such as the age of the catenary, the supply voltage, the quality of the pantograph, weather conditions...etc.

However, we think that somehow the bandwidth of the GSM$\mathrm{R}$ antenna could affect the measurement characteristics of the different transients, since the GSM-R antenna used along all the measurement campaigns laid the same for all the collected transient disturbances.
Moreover, a note of caution is in order here since the different statistical laws and parameters presented below do not narrowly illustrating the real statistical distributions of the studied parameters. Representative laws of the different characteristics of the transient events could be obtained with a flock of measurement campaigns performed with different configurations and operating conditions. However, our main objective in this part remains the presentation and the feasibility verification of a new method to characterize the immunity of the GSM-R system.

\subsection{Rise Time and duration}

For each transient signal collected, the rise time $\boldsymbol{R} \boldsymbol{T}$ and the time duration $\boldsymbol{T D}$ were calculated using the definitions of the European standard 61000-4-4, specified previously.

The experimental $\boldsymbol{R} \boldsymbol{T}$ and $\boldsymbol{T D}$ probability density functions (PDFs) of the collected transients are presented in Figure 4 by the bar diagrams. From the experimental PDFs of the rise time and time duration of the transients, empirical PDFs approaching the experimental ones are presented by the dashed lines in Figure 4.
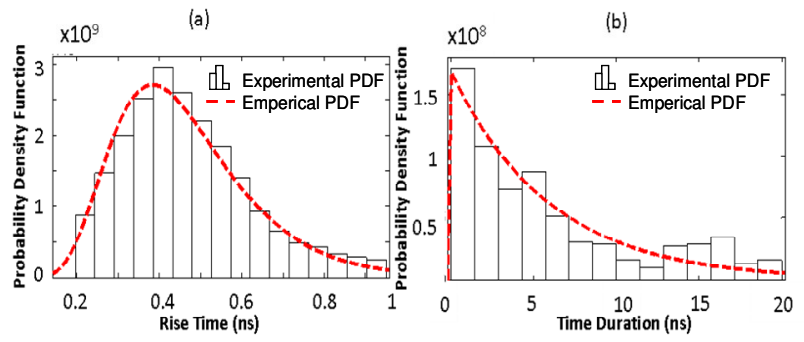

Figure 4: Experimental and empirical distributions of the Rise Time (a) and duration (b) of the transient events in ns

The empirical distribution of $\boldsymbol{R T}$ follows a lognormal law, where the PDF is given by (1).

$f(R T / \mu, \sigma)=\frac{1}{R T \sigma \sqrt{2 \pi}} \exp \left(-\frac{(\ln (R T)-\mu)^{2}}{2 \sigma^{2}}\right)$

where $\boldsymbol{\mu}=-21.55$ and $\boldsymbol{\sigma}=0.36, \boldsymbol{\mu}$ and $\boldsymbol{\sigma}$ are the distribution parameters and are estimated by means of the maximum likelihood estimation method with $95 \%$ confidence intervals. The results show that the rise times are inferior to $1 \mathrm{~ns}$ and the more common rise time is $0.4 \mathrm{~ns}$.

The empirical distribution of $\boldsymbol{T D}$ follows an exponential law, where the PDF is given by (2).

$$
f(T D / \lambda)=\frac{1}{\lambda} \exp \left(-\frac{T D}{\lambda}\right)
$$

where $\lambda=5.93 .10-9$ is estimated according to the maximum likelihood estimation method with $95 \%$ confidence interval. The results show that the transient durations are systematically inferior to $20 \mathrm{~ns}$ and mean time duration is about $5 \mathrm{~ns}$. We notice that, compared to the duration of the 
transmission of one GSM-R bit, the mean time duration of the transients is extremely short $(5 \mathrm{~ns}<3.7 \mu \mathrm{s})$.

Supplementary measurement campaigns (not presented in this paper) carried out under other operating conditions (speed, locomotive model, train path, weather conditions, train length...), showed that the distribution of the rise time and time duration are constant, whatever the operating conditions. However, we have to keep in mind that the distributions of RT and TD are partially related to the frequency bandwidth of the GSM-R antenna which can limit the transient EM events' spectrums.

Consequently, by employing a reception antenna with different bandwidths, different distribution would be probably obtained.

We have to point out the fact that a Goodness of Fit (GOF) tests based on the Kolmogorov-Smirnov method [7] were performed to identify the validity of the presented laws. The results showed that the experimental distributions of TD and RT could be well fitted with the use of other distribution laws that we avoided the use in this paper.

\subsection{Time interval between two successive transients}

The time interval $(\boldsymbol{T I})$ is a significant parameter for the study of the immunity of the GSM-R system since the interpretation of a GSM-R received bit could be spoiled when combined with an EM transient event.

On the one hand, if the time intervals between successive transients are short, that means that several bits within a GSM-R burst can be affected,

On the other hand, if the time intervals are long, very few bits can be affected during a single burst. In that case, the transients cannot affect the information transmission.

Knowing that each user employs a GSM-R frequency channel one eighth of the time and over periods of $577 \mu$ s, we aimed to measure the EM noise on board moving trains along comparable time durations, with a digital oscilloscope connected to the GSM-R antenna. Our objective being to detect the number of transient events covering the GSM-R frequency bands, and knowing that the bandwidth of the GSM-R antenna is limited to $1 \mathrm{GHz}$, the sampling frequency of the oscilloscope was fixed at $5 \mathrm{GHz}$. However, with such a sampling frequency, the memory depth (2.106 mega points) of the oscilloscope used limited the window to $400 \mu \mathrm{s}$. The results presented below are then based on $400 \mu \mathrm{s}$ time windows.

During a period of 25 minutes (1,500 seconds), we recorded 1,568 files (or 1,568 time windows of $400 \mu \mathrm{s}$ ) and the time required to record the data of a $400 \mu \mathrm{s}$ window on the oscilloscope hard disk is rather less than 1 second. Thus, the oscilloscope was continually activated by the presence of transient signals over the entire measurement period. Hence, we can suppose that the distribution of the time intervals obtained from the 1,568 time windows is representative of the entire duration measurement ( 25 minutes).

To obtain the time interval between the transients, the first step is to recognize the transient events in the $400 \mu$ s time window. The second step consists in calculating the time difference between the consecutive transient events in each window. In order to detect the transient events, we developed an algorithm allowing $3 \mu \mathrm{s}$ resolution time, which approximately corresponds to the duration of one GSM-R bit. Thus, the number of transient events detected will correspond to the potential number of the disturbed GSM-R bits. This value of $3 \mu$ s then limits to the minimum time interval that we can obtain.

Figure 5 gives examples of a collected time windows. The analysis of the recorded files shows that 1,030 files contain only one transient event in $400 \mu$ s. So, they cannot allow us to extract time intervals. 40 supplementary files were not employed due to their containing a high level of permanent noise probably coming from the GSM public transmitter nearby. Indeed, a relatively high permanent noise (illustrated in Figure 5) can hide the transient EM events and does not permit us to extract the correct number of the transient disturbances which occurred during the analysis time window. Finally, only 498 files containing a minimum of two transient events were employed. From these files, we extracted 1,121 time intervals less than $400 \mu \mathrm{s}$.
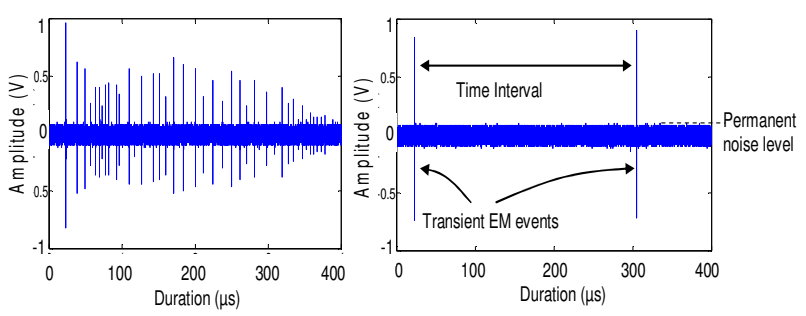

Figure 5: Examples of measured files over $400 \mu$ s time windows

The bars diagram in Figure 6 shows the experimental PDF of the 1,121 collected time intervals. According to the experimental PDF of the time intervals, empirical PDF approaching the experimental one is presented by the dashed line in Figure 6.

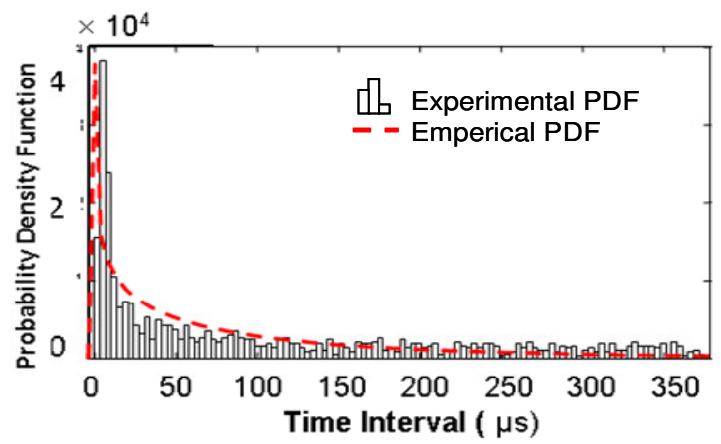

Figure 6: Experimental and empirical probability density functions (PDF) of the time intervals between two consecutive transient events 
The empirical distribution of $\boldsymbol{T I}$ follows an Weibull law, where the PDF is given by (3).

$$
f(T I / a, b)=b \cdot a^{-b} \cdot T I^{b-1} \cdot \exp -\left(\frac{T I}{a}\right)^{b} \quad \text { for } T I>
$$

where $\boldsymbol{a}=9.2210-5$ is the scale parameter and $\boldsymbol{b}=0.77$ is the shape parameter. The parameters $(\boldsymbol{a}$, and $\boldsymbol{b})$ of the distribution are estimated by means of the maximum likelihood method with $95 \%$ confidence intervals.

The experimental PDF of the time intervals less than $400 \mu \mathrm{s}$ corresponds perfectly to the Weibull's PDF presented above.

In Figure 6, we noticed that the time intervals are mainly inferior to $17 \mu \mathrm{s}$. Assuming that each transient interference can affect the interpretation of one transmitted bit, transient disturbances which occur with $17 \mu$ s time intervals could cause a $20 \%$ bit error rate.

Besides, we have to keep in mind that this distribution does not take into account the time intervals higher than $400 \mu \mathrm{s}$. Indeed, $48 \%$ of the time intervals collected are higher than $400 \mu \mathrm{s}$. We will have to improve the method in order to include these time intervals at the time of the generation of the transient scenarios.

\subsection{Peak Amplitude of the transients}

With the same methodology employed for the extraction of the PDF of $\boldsymbol{R} \boldsymbol{T}, \boldsymbol{T D}$, and $\boldsymbol{T I}$ we studied the PDF of the peak amplitudes $\boldsymbol{A}$ of the transient events. The analysis showed that $\boldsymbol{A}$ follows the Generalized Extreme Value (GEV) distribution law where the PDF is expressed by (4).

$$
f(A ; \mu, \sigma, \zeta)=\frac{1}{\sigma}\left[1+\zeta\left(\frac{A-\mu}{\sigma}\right)\right]^{1 / \zeta^{-1}} \exp \left\{-\left[1+\zeta\left(\frac{A-\mu}{\sigma}\right)\right]^{-1 / \zeta}\right\}
$$

where $\boldsymbol{A}$ is the peak amplitude, $\boldsymbol{\mu}=0.06$ is the location parameter, $\sigma=0.02$ is the scale parameter, and $\xi=0.37$ is the shape parameter. $\boldsymbol{\mu}, \boldsymbol{\sigma}$, and $\boldsymbol{\xi}$ are estimated by means of the maximum likelihood method with $95 \%$ confidence interval.

However, under other measurement configuration, mainly the position of the GSM-R antenna, the distribution law parameters of the amplitude can vary regarding to the position gap between the GSM-R antenna and the pantograph. Thus, according to the position of the antenna, the amplitude distribution law parameters must be adjusted to fit the corresponding experimental distribution of the transients' peak amplitudes.

\section{Typical transient disturbances collected on the train and comparison with the transient test signal definition applied in the Standard EN 61000-4-4}

Nowadays, the immunity tests of the electronic systems against transient EM disturbances are performed respecting the recommendations given in the European Standard EN 61000-4-4. A comparison between the time characteristics of the transient events defined in this standard and the typical time characteristics obtained with GSM-R antenna on board moving train is presented in Table. 1.

The time characteristics of the transients described in the EN 61000-4-4 standard are widely higher than the ones received by the GSM-R antenna on board the train.

Previous studies [8] showed that the EM noise amplitude generated by a transient event in the frequency band of the GSM-R system is related to time characteristics ( $R \boldsymbol{T}$ and $\boldsymbol{T D})$ of this transient event. In particular, the test signals defined in the EN 61000-4-4 do not cover the frequency bands of the GSM-R while the transient disturbances observed on board train systematically cover the GSM-R channels.

\begin{tabular}{|l|c|c|c|}
\hline & $\begin{array}{c}\text { EN 61000-4-4 } \\
(1)\end{array}$ & On train (2) & Comparison \\
\hline $\begin{array}{l}\text { Rise } \\
\text { Time }\end{array}$ & $5 \mathrm{~ns} \pm 30 \%$ & $0.4 \mathrm{~ns}$ & $\boldsymbol{R T}(1)>>\boldsymbol{R T}(2)$ \\
\hline Duration & $50 \mathrm{~ns} \pm 30 \%$ & $5 \mathrm{~ns}$ & $\boldsymbol{T D}(1)>>\boldsymbol{T D}(2)$ \\
\hline $\begin{array}{l}\text { Time } \\
\text { Interval }\end{array}$ & $\begin{array}{c}0.2 \mathrm{~ms} \text { and } \\
0.4 \mathrm{~ms}\end{array}$ & $25 \mu \mathrm{s}$ & $\boldsymbol{T I}(1)>>\boldsymbol{T I}(2)$ \\
\hline
\end{tabular}

Table 1: Comparison between the time characteristics of the transient disturbances observed on board the train and the ones applied in the standard EN 61000-4-4

Besides, intuitively we can think that the more short the time interval between two successive transient events, the more the system's immunity is affected. Regarding to the time interval between two successive transients in the standard, we notice that with a time interval included between $0.2 \mathrm{~ms}$ or $0.4 \mathrm{~ms}$, a maximum of one bit can be affected during a $577 \mu$ s period (corresponding to the GSM-R time slot). Nevertheless, in practise, we observed that under certain operating conditions, a typical time interval can be $25 \mu \mathrm{s}$. In that case, 23 bits are potentially affected by the transient disturbances. Hence, the immunity tests based on the EN 61000-4-4 are not adapted for the immunity's study of the GSM-R system and are not representative of real EM risks induced by the transient disturbances on board trains. So, a relevant immunity test needs the generation of a transient events scenario similar to that observed on board a moving train. In the next part we propose to generate a transient events scenario statistically similar to that observed on board a moving train.

\section{Generation of the transient disturbance scenarios}

To generate transient scenarios with time and amplitude characteristics statistically similar to that observed on board a moving train, we assumed that these characteristics are statistically independent.

The first step of the generation process is to generate a table with four lines and $\mathrm{N}$ columns. Each line corresponds respectively to the rise times, time durations, peak amplitudes, and time interval of one transient event.

$\mathrm{N}$ corresponds to the number of the transients in one scenario. The line's values are generated with MATLAB and have a 
corresponding statistical distribution similar to that obtained above.

If $\mathrm{K}$ is the rank of apparition of a transient event in the generated scenario, then its different characteristics correspond to the values of the Kth column.

The general expression of the observed transient signals can be theoretically defined by (5).

$V(t)=A\left(\exp \left(-\frac{t}{F T}\right)-\exp \left(-\frac{t}{R T}\right)\right) \gamma(t)$

$\boldsymbol{V}(\boldsymbol{t})$ is the transient signal voltage. $\boldsymbol{A}$ is the peak amplitude, $\boldsymbol{R T}$ is the rise time, $\boldsymbol{F T}$ is the fall time, and the causality of the signal is given by the echelon function $\gamma$.

According to the $\boldsymbol{T D}$ and the $\boldsymbol{R} \boldsymbol{T}$ of the signal, the $\boldsymbol{F T}$ is computed automatically [9]. Once all the $\mathrm{N}$ transient events generated, the concatenation of the transients is performed with MATLAB to generate the transient noise sequence.

Figure 7 presents a transient events scenario where the distributions of the time characteristics of the transient events follow the probability density functions given above. Figure 7.a gives one scenario of $4 \mathrm{~ms}$ time long where 30 transient events are observed. Figure $7 \mathrm{~b}$ is a Zoom-In of Figure 7a showing 10 transient events occurring along a $577 \mu$ s time duration corresponding to one GSM-R time slot.

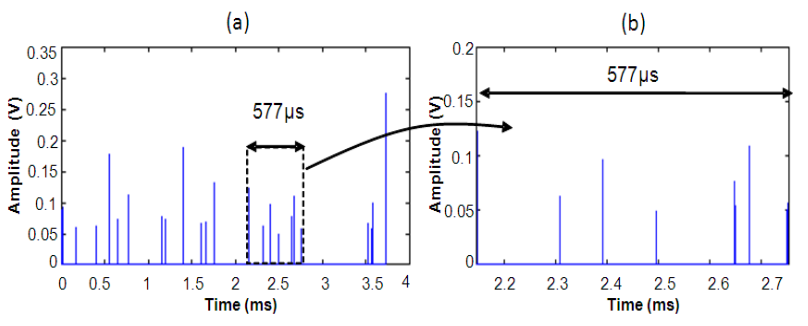

Figure 7: Transient events scenario statistically similar to the ones observed on board moving train with a GSM-R antenna

It is, therefore, possible to identify sequences of EM transient interferences closer to reality than the electromagnetic noise conditions described in the 61000-4-4 standard. These results allow us to consider two major perspectives. Firstly, we plan to work on the generation of electromagnetic noise sequences (using arbitrary signal generators) within electromagnetic test facilities in order to test the immunity of GSM-R transmission given these conditions of transient EM noise. Secondly, the simulation chains of communication systems can generally simulate the behaviour of the system in the presence of Gaussian distribution EM noise. We might, therefore, consider undertaking these simulations with statistical distributions of EM noise more representative of certain areas.

\section{Conclusions}

A statistical study of the time characteristics of the transient events observed through a GSM-R antenna fixed on the roof of a moving train has been presented in this paper. Experimental and empirical distributions of each time parameter of the transient events have been presented and compared. These results show that the definitions of the transient disturbances currently employed in the standard 61000-4-4 are significantly different from the transient disturbances observed on the train roof. According to the empirical distributions obtained, an EM transient noise scenario statistically similar to the experimental one was generated. Thus, we envisage to carry out relevant immunity tests of the GSM-R system in a laboratory context.

Currently, we are working on an adapted methodology allowing the generation of the transient EM noise scenario taking into account the possible dependency between the time characteristics of the transient events, in order to perform relevant GSM-R immunity tests. A new on-board methodology allowing the measurement of time interval higher than $400 \mu \mathrm{s}$ has also to be developed.

If this study would lead to standardized recommendations for the study of the EM immunity of such a digital communication system, an exhaustive study of the transient EM events characteristics has to be planned under different operating conditions and configurations.

\section{Acknowledgements}

This work was carried out within the framework of the RAILCOM project and benefited of the financial support of the PCRD 6. The measurement campaigns would not been able to be carried out without the contribution of the SNCF.

\section{References}

1. M. Uhlirz, Concept of a GSM-based Communication System for High-speed Trains, IEEE 44th Vehicular Technology Conference, Stockholm, Sweden 1994.

2. R. Steele, C. Lee, P. Gould, GSM, cdmaOne and $3 G$ Systems, John Wiley \& Sons Ltd, 2001

3. European Standard EN 61000-4-4 : Electromagnetic Compatibility Part 4: Testing and measurement techniques-Electrical fast transient/burst immunity test, May 2001.

4. European Standard ETSI EN 300386, Electromagnetic compatibility and Radio spectrum Matters (ERM); telecommunication network equipment, ElectroMagnetic Compatibility (EMC), requirements", March 2000.

5. R. Adriano, N. Ben Slimen, V. Deniau, M. Berbineau, P. Massy, Prediction of the BER on the GSM-R communications provided by the EM transient disturbances in the railway environment, Electromagnetic Compatibility Europe 2008, Hambourg-Germany, September 2008 
6. NIST/SEMATECH 'e-Handbook of Statistical Methods', http://www.itl.nist.gov/div898/handbook/, January 2003

7. F. J. Massey, _The Kolmogorov-Smirnov test for goodness of _t, Journal of the American Statistical Association, vol. 46, pp. 68_78, 1951.

8. V. Deniau, J. Rioult, N. Ben Slimen, H. Ouaddi, N. Dubalen, Railcom consortium, Characterisation of the electromagnetic disturbances received by GSM-R antennas in usual railway operating conditions, EMC Europe Workshop, Paris-France, May 2007

9. M. Camp, H. Garbe, Parameter estimation of double exponential pulses (EMP, UWB) with Least Squares and Nelder Mead algorithm, IEEE Transactions on Electromagnetic Compatibility, Vol. 46, No. 4, pp. 675-678, November 2004

\section{Figures and Table Captions}

Figure 1: Time characteristics of one GSM-R Burst

Figure 2: Time characteristics of the transient events Figure 3: Measurement configuration

Figure 4: Experimental and empirical distributions of the Rise Time (a) and duration (b) of the transient events in ns

Figure 5: Example of measured file with two transient events in a $400 \mu$ s time window

Figure 6: Experimental and empirical distributions of the time intervals between two consecutive transient events

Figure 7: Transient events scenario statistically similar to the ones observed on board moving train with a GSM-R antenna

Table 1: Time characteristics of the transients observed on board the train and the ones given in the EN 61000-4-4 